\title{
GÉNERO Y DIFERENCIAS COGNITIVAS \\ EN LA SOLUCIÓN DE PROBLEMAS \\ DE RAZONAMIENTO ESPACIAL
}

Martha R. Rubio S*

\begin{abstract}
This paper aims to present that cognitive differences in spatial reasoning are not only due to biological component (female or male genres), but also to the learning enviroment in which individuals are involved. This component affects deeply this kind of reasoning. The statistics analysis of data collected in this investigation by using a computer program "Play and discover: spatial reasoning and design", concluded that when male and female individuals are engaged in reasoning problems under the same learning environments, efflcay and efficiency differences have a tendency fo diminish.
\end{abstract}

\section{RESUMEN}

Este artículo muestra las diferencias cognitivas existentes en cuanto al razonamiento espacial, no sólo dadas por el componente biológico (hombre, mujer), sino también el ambiente en el cual se desarrollan los sujetos el cual incide de manera categórica. Los datos recogidos para este trabajo a través del programa de computador denominado "JUEGUE Y DESCUBRA: Razonamiento Espacial y Diseño", dejó ver luego del análisis estadístico realizado, que cuando los géneros masculino y femenino solucionan problemas de R.E en igualdad de condiciones, las diferencias en la eficacia y la eficiencia tienden a disminuir.

En este trabajo de tesis no se pretende demostrar que entre hombres y mujeres no existen diferencias cognitivas, se buscó indagar si entre jóvenes de colegio mixto existían diferencias en cuanto a la eficacia y eficiencia cuando solucionaban problemas de razonamiento espacial basados en computador.

La tesis para acceder al título de Master tomó como base las investigaciones que en el Centro de Informática de la Universidad Pedagógica (CIDUP) se adelantaron sobre la temática de diseño y razonamiento espacial. En la revisión realizada a las investigaciones me di cuenta que la variable género no había sido tenida en cuenta en ninguna de las tres investigaciones; al estar esta opción libre a los datos recogidos en la investigación sobre Incidencia de Estrategias y Juicios de Metamemoria en Problemas de Razonamiento Espacial, decidimos junto con el director de la tesis aplicar un tratamiento estadístico de regresión múltiple para observar el comportamiento de la variable género de manera independiente con el fin de determinar si el género incidía de manera significativa en la Eficacia y la Eficiencia de este grupo de adolescentes cuando solucionaban problemas de razonamiento espacial basados en computador.

\footnotetext{
Investigadora Centro de Informática. Línea de Aprendizaje Autónomo. Artículo basado en la tesis de Maestría en Tecnologías de la información aplicadas a la educación.
} 
Así, este trabajo se nutrió de los componentes teóricos sobre diferencias entre géneros, análisis de procesos cognitivos (ver Metacognición y razonamiento espacial en juegos de computador, Maldonado et al, 1999), y el desarrollo de software educativo. El componente informático se diseño a partir de los planteamientos de Rudolf Arheim sobre Arte y Percepción visual (Equilibrio). El soporte informático no solo sirvió para que los jóvenes jugaran, este también se encargaba de hacer la recolección de todos los factores que se consideraban importantes para realizar el análisis estadístico (como en toda investigación estoy consiente que existen aspectos que no se consideraron aquí) como: tiempo total de desarrollo del juego (problema), tiempo promedio, cantidad de eventos invertidos para concluir el juego (entendiendo como evento los click realizados con el mouse) y cada una de las acciones que el sujeto realizaba a través de mouse para llegar a la solución. La recolección que se hizo por medio del mismo software además de permitir analizar estadísticamente los datos también facilita replicar los procesos que los sujetes siguieron para desarrollar los cuatro problemas que se analizaron en este trabajo.

Es necesario aclarar a que se hace referencia cuando se habla de Eficacia y Eficiencia. Definida eficacia como la relación entre eventos exitosos y el total de eventos invertidos en la solución del problema y eficiencia como la relación entre eventos exitosos y la cantidad de tiempo requerido para resolverlo.

En el aprendizaje de los juegos se consideraron tres etapa para observar el desarrollo de la habilidad de los sujetos en la medida que solucionaban el juego, esto en coherencia con la curva de aprendizaje planteada en los estudios clásicos de la psicología, donde se muestra que existe un mejoramiento más acelerado en el inicio de la solución de un problema que al final (Restle y Greeno, 1970). Las etapas de aprendizaje se determinaron de la siguiente manera:

Etapa de descubrimiento: Se caracteriza por ser el momento en el cual el sujeto inicia su adaptación al sistema, explora y analiza el espacio del problema e intenta construir una estrategia de solución.

Etapa de consolidación I: En este estado el sujeto prueba si la estrategia desarrollada es valida o si debe readaptarla.

Etapa de consolidación II: Como se puede inferir, en este momento el sujeto ya consolida la manera de solucionar el problema de forma eficaz y eficiente.

Es de anotar que los cuatro problemas a solucionar estaban programados a manera de juegos: 1) líneas en equilibrio, 2) líneas al azar, 3) agujeros en equilibrio y 4) agujeros al azar. Cada uno de los juegos debían solucionarlo tres veces, para poder observar qué pasaba con la eficacia y la eficiencia en cada una de las etapas de aprendizaje en relación con la variable independiente, Género.

En cuanto al sustento teórico relacionado con las diferencias cognitivas entre géneros, las investigaciones están desarrolladas desde la neuropsicología, aquí se plantea que las diferencias entre los géneros no sólo está basada en la capacidad reproductora o fenotípica, también han encontrado que las hormonas sexuales condicionan la organización cerebral en una etapa precoz de la vida; y así desde los comienzos, el ambiente actúa sobre los cerebros que presentan un sistema de ordenación distinto según se trate de la niña o del niño (Kimura, 1992).

El planteamiento fuerte de los investigadores que han trabajado sobre esta temática esta relacionado con la manera como solucionan problemas hombres y mujeres, no con el 
propósito de buscar cuál de los géneros resulta ser más inteligente o puede dominar, sino con el ánimo de dejar ver cómo las diferentes ocupaciones y preferencias profesionales se orientan según el ordenamiento cerebral de cada género.

En lo que hace referencia a las investigaciones de corte neuropsicológico se ha encontrado que las mujeres tienden a rendir mejor en tareas que impliquen la habilidad verbal, la memoria, la motricidad fina y cálculo aritmético, mientras que los hombres han demostrado tener mejores resultados en cuanto a lo que implica la habilidad espacial, el razonamiento matemático y motricidad gruesa. Con esto no se está diciendo que alguno de los dos géneros tenga un coeficiente intelectual mayor porque desarrolle más habilidades propias de uno $u$ otro hemisferio, simplemente se plantea que existen diferencias de orden biológico que de una u otra forma se acentúan con el medio ambiente en el que se desenvuelve cada sujeto.

Hasta aquí todo parece claro en relación a la neuropsicología, pero en la pedagogía son muy pocos los estudios desarrollados y los existentes están enfocados al análisis de la habilidad matemática, y no con la habilidad espacial y menos aún relacionando con los amblen-tos informáticos como soportes para la solución de problemas de razonamiento espacial. Camila P. Benbow en su estudio Diferencias sexuales en la habilidad de razonamiento matemática en preadolescentes intelectualmente talentosos: Su naturaleza, efectos y posibles causas", concluye que una capacidad matemática elevada se apoya en un componente biológico significativo. En el grupo investigado por Bebow el componente sexo presenta gran incidencia en cuanto a la capacidad de razonamiento matemático favoreciendo de manera extrema a los varones, pues, aventajan a las mujeres en una relación de 13 a 1 en los problemas solucionados. Ella sostiene que las diferencias halladas no tienen una explicación fácil desde el componente social. Este argumento no se puede rebatir con una sola investigación pero los resultados encontrados en este trabajo de tesis muestra que de alguna manera el medio en el cual se educan los sujetos si incide en los procesos cognitivos y de solución de problemas como se ve en las ilustraciones más adelante.

La evidencia que apoya la idea de la existencia de diferencias sexuales significativas en la organización cerebral deriva principalmente de los estudios de individuos normales y de los estudios de lateralidad (pacientes neurológicos). En individuos normales MacCoby y Jacklin (1974), encontraron 4 diferencias sexuales importantes: 1) Las mujeres tienen mayor habilidad verbal que los varones y realizan mejor las tareas en las que interviene la recepción y producción del lenguaje. 2) En tanto, los varones son superiores en la habilidad visoespacial. Así, en los tests de recuerdo y detección de formas, de rotación mental de las figuras bi o tri-dimensionales, de geometría, o de aprendizaje de laberintos, los hombres en promedio son mejores que las mujeres. Al igual que con la ventaja verbal de las mujeres, la ventaja espacial de los hombres no es absoluta. 3) Los hombres son superiores en cuanto a la habilidad matemática. Aunque los dos géneros adquieren de forma similar los conceptos matemáticos y el dominio aritmético; puede deberse según los autores a la mayor cantidad de entrenamiento matemático al que se someten los hombres. 4) Los hombres son más agresivos físicamente que las mujeres.

Aunque las diferencias sexuales descritas por MacCoby y Jacklin han sido citadas a menudo como una ventaja espacial para los hombres y una ventaja verbal para las mujeres, sin embargo esta dicotomía es demasiado simple, pues no se pueden etiquetar las diferencias cognitivas sólo como verbal y espacial, asunto sobre el cual es necesario investigar más. Pero es importante tener en cuenta que las diferencias presentadas entre 
los géneros también pueden ser resultado de variables culturales y/o ambientales, las cuales deben considerarse al llevar a cabo una investigación.

Si las diferencias descritas anteriormente son concluyentes o validas, podría esperarse encontrar una diferencia entre los géneros en la distribución de las funciones verbales y espaciales de los hemisferios en los estudios de lateralidad.

Las diferencias en las habilidades, destrezas y comportamientos (especialmente relacionadas con las aptitudes cognitivas) tales como el rendimiento académico, la aparición del lenguaje, la proporción de estudiantes según el sexo en distintas carreras y oficios, entre muchos otros, muestran claramente distinciones entre hombres y mujeres (Pueyo, 1996). En la medida en que los psicólogos han estudiado más analítica y exhaustivamente estas diferencias, pretendiendo encontrar evidencias empíricas, basadas en las diferencias de capacidades o aptitudes cognitivas de los hombres y las mujeres (evidencias sólidas de las mismas), lo que parecía nítido y patente se va disolviendo hasta desaparecer, del mismo modo como sucede al mirar un cuadro pintado con la técnica del puntillismo. Estos cuadros desde una determinada distancia, nos muestran claramente un paisaje o una figura, pero si nos acercamos al lienzo, solamente vemos un conjunto más o menos informe de puntos de colores y el paisaje original o la figura se ha difuminado o desaparecido. Según Pueyo, algo similar pasa cuando se investigan las diferencias sexuales en las capacidades cognitivas. Lo que parece obvio en el comportamiento social o académico se diluye al buscar su causa en una distribución diferencial de las capacidades cognitivas entre los sexos.

\section{RESULTADOS}

¿Existen diferencias en el rendimiento cognitivo dadas directamente a diferencias de sexo en las capacidades intelectuales? A pesar de que las diferencias entre sexos, en rendimiento cognitivo son de pequeña magnitud y tienden a variar en función de las muestras que se estudian, unas cuantas diferencias en promedio se encuentran en ciertas capacidades (Maccoby y Jacklin, 1974). Las que más interés tienen, por su aparición consistente, son las conocidas en las capacidades verbales, numérico-matemáticas y espaciales.

Teniendo en cuenta que el análisis de este trabajo se hizo desde la perspectiva pedagógica para determinar si existía diferencia significativa en cuanto a la eficacia y eficiencia cuando solucionan problemas de razonamiento espacial basados en ambientes computarizados hombres y mujeres. Se encontró que las diferencias son mínimas y que posiblemente de manera significativa el medio en el cual se desarrolla cognitivamente un sujeto influye de forma determinante.

A continuación se muestran las ilustraciones de los datos analizados en los cuales podemos inferir que si bien hombres y mujeres solucionan de manera diferente los problemas, en cuanto a la eficacia y la eficiencia, la diferencia es poca. Con esto no se pretende demostrar que no existen diferencias en el nivel cognitivo sino que no, es posible generalizar que los hombres siempre serán superiores en el aspecto del razonamiento espacial en relación con las mujeres, por lo menos en los problemas aquí analizados.

En esta gráfica observamos que ambos géneros en el primer módulo presentan procesos de solución similares, luego en el módulo dos y tres la línea que representa a los hombres tiende a superar a la que representa a las mujeres, pero estadísticamente no se 
encuentran diferencias significativas entre los dos; en el último módulo tienden a igualarse nuevamente los procesos.

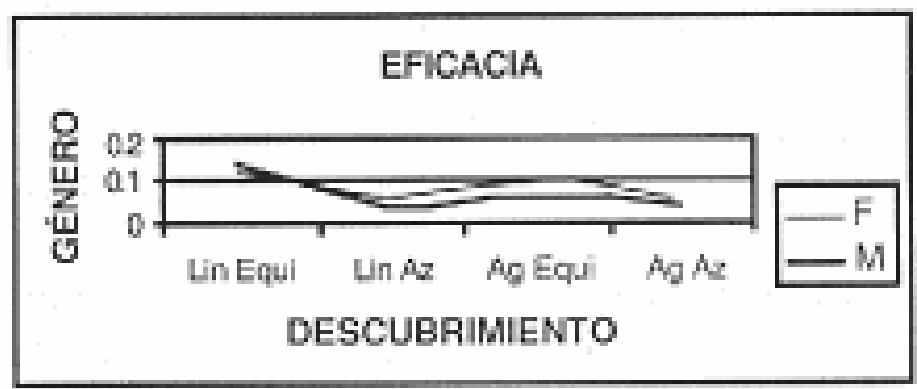

llustración 1. Muestra el proceso en la solución de los cuatro módulos en la etapa de Descubrimiento realizada por ambos géneros.

En esta gráfica observamos que los géneros en el primer módulo difieren muy poco, pero, en los tres módulos restan-tos no se presentan diferencias, tal como lo muestra las líneas en el esquema.

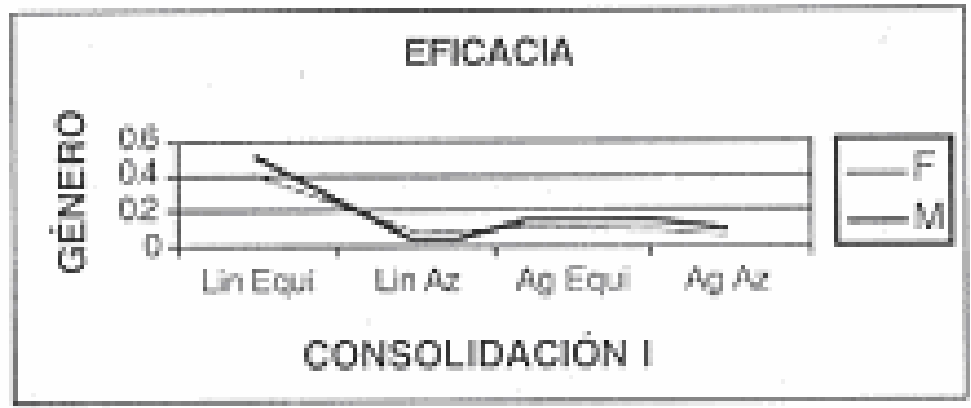

llustración 2. Muestra el proceso de solución en los cuatro módulos en la etapa de Consolidación 1.

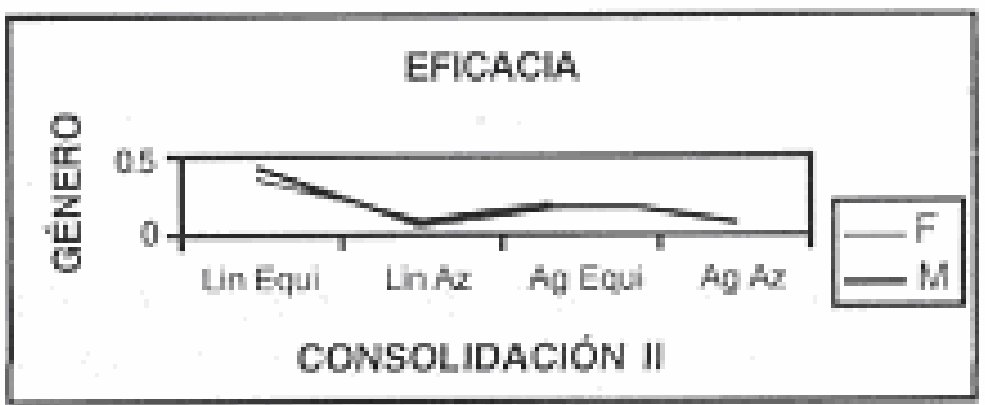

Ilustración 3. Muestra el proceso en la solución de los cuatro módulos en la etapa de Consolidación II realizada por ambos géneros.

La gráfica muestra que no hay diferencias en esta última etapa del proceso.

Las anteriores gráficas muestran la evolución de los dos géneros durante el proceso de solución de los problemas en cuanto a la variable dependiente eficacia.

Ahora, las siguientes ilustraciones muestran el comportamiento de la variable dependiente eficiencia contra la variable independiente género. 


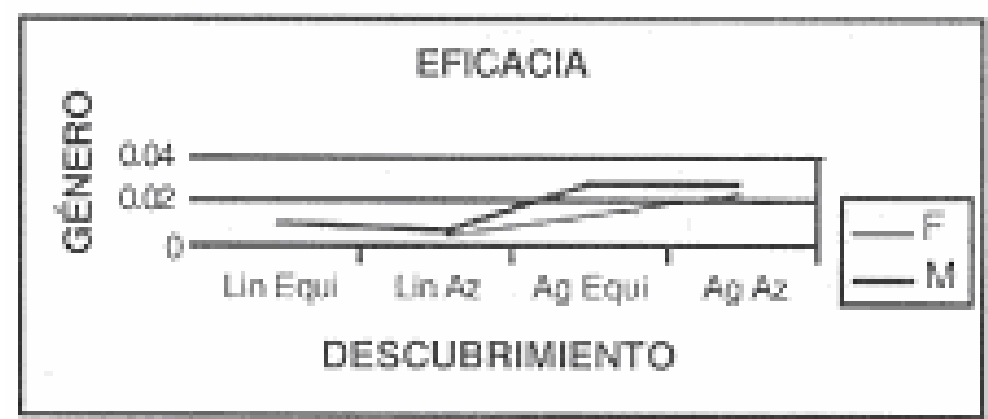

Ilustración 4. Muestra el proceso en lasolución de los cuatro módulos en la etapa de Descubrimiento.

La graficación de los datos muestra una tendencia de los géneros a mantenerse en esta primera etapa, donde los niveles de significación son similares.

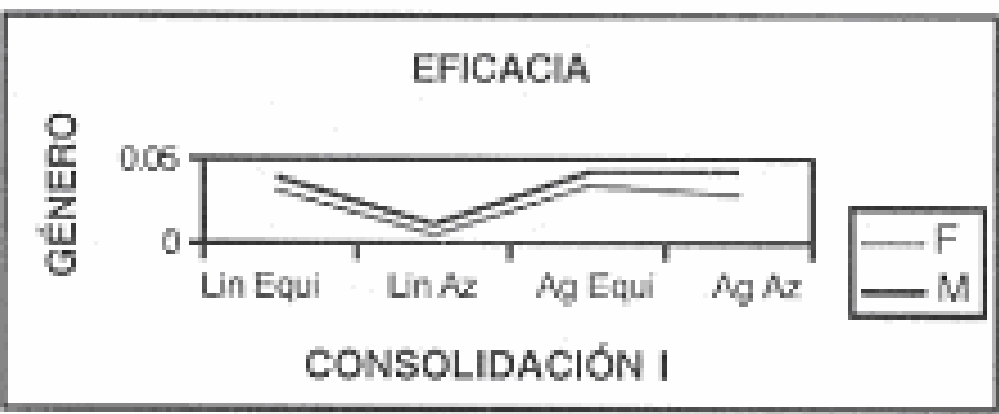

Ilustración 5. Muestra el proceso de solución de los cuatro módulos en la etapa de Consolidación 1.

La gráfica permite observar que los datos sobre los dos géneros no sobrepasan el nivel de significación, por lo tanto el nivel de rendimiento es estadísticamente igual pese a la leve superioridad de las mujeres.

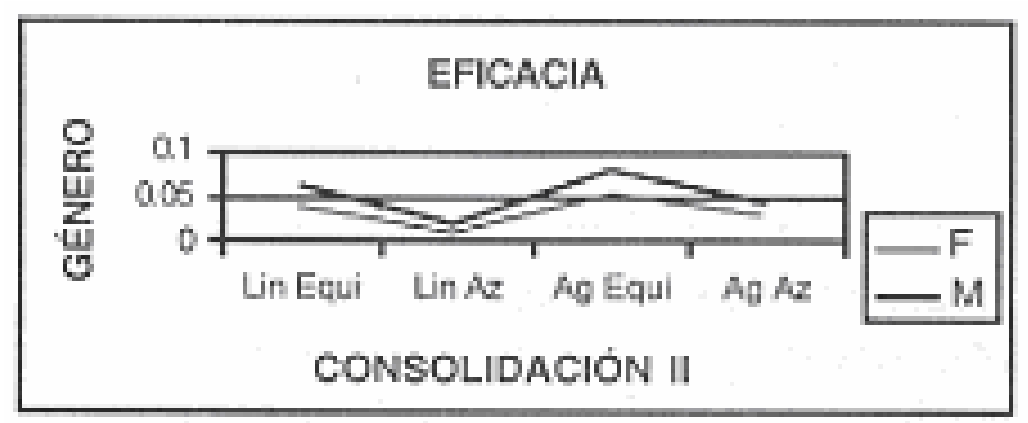

Ilustración 6. Muestra el proceso en la solución de los cuatro módulos en la etapa de Consolidación II. 
En la gráfica se observa una mayor tendencia de la línea que representa los datos de las mujeres a mantenerse por debajo del 0.05 , mientras la línea que representa a los hombres supera en el tercer módulo el nivel mínimo de significación.

\section{REFERENCIAS BIBLIOGRÁFICAS}

Arheim, R. (1997). Arte y percepción visual. Madrid: Alianza Editorial, Primera Edición, 1971.

Benbow, C. P. (1988). "Sex differences in mathematical reasong ability in intellectually talented preadolescents: Their nature, effects, and possible causes". En: Behavioral and Brain Sciences, 11, 169-232.

Kimura, Doreen. Sex and Cognition. Instituto of Technology. Massachusetts: Cambridge, 1999.

Kimura, Doreen (1992). "Cerebro de varón y cerebro de mujer". En: Investigación y ciencia. Nov. 77-84.

Kolb, Bryan \& Whishaw, lan. Fundamentos de neuropsicología humana. Universidad de Lethbridge. De. Labor SA. 1986.

Maldonado, L., Rubio, M., Sanabria, L., Fonseca, O., Macias, D., Ibañez, J., Ortega, N. Metamemoria y razonamiento espacial en juegos de Computador. Investigación convenio U.P.N e DEP. 1999.

Pueyo, Antonio A. Manual de psicología diferencial. Madrid: Mac Graw Hill, 1996.

Restle, F. and Greeno, J. G. (1970). Intruduction to Mathematical Psychology. Headig. Massachusetts: Addison-Wsly Publishing Company. 